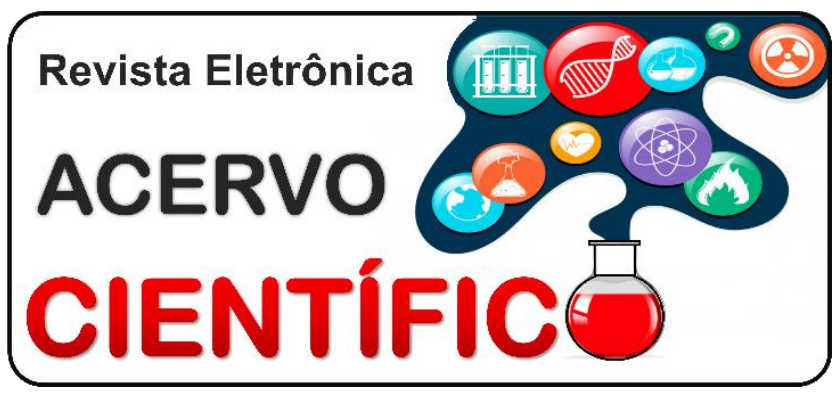

\section{REVISÃO BIBLIOGRÁFICA}

Recebido em: 12/2020

Aceito em: $1 / 2021$

Publicado em: 2/2021

\title{
A relação da microbiota intestinal na síndrome do intestino irritável
}

\author{
The relationship of intestinal microbiota in irritable bowel syndrome
}

La relación de la microbiota intestinal en el síndrome del intestino irritable

Mayra Loures de Oliveira ${ }^{1 *}$, Nestor Augusto Avelino Leite ${ }^{2}$, Agnes Zanotto Manoel ${ }^{3}$, Bárbara Gonçalves Flor ${ }^{4}$, Bruna de Sousa Silva ${ }^{5}$, Dandara Lucena Medeiros Madeiro ${ }^{6}$, Julia Perito Alfredo ${ }^{7}$, Maria Cecília Baratela ${ }^{8}$, Nicole Hana Romanisio ${ }^{9}$, Poliana Zanotto Manoel ${ }^{3}$.

Resumo: Este artigo de revisão buscou discutir acerca da relação da microbiota intestinal na síndrome do intestino irritável, a qual é uma das doenças do trato gastrointestinal mais comum no mundo e leva a alterações dos hábitos intestinais gerando dor e desconforto abdominal. Sua fisiopatologia ainda não é bem estabelecida, entretanto, estudos recentes demonstram que a alteração da microbiota intestinal pode ser a desencadeadora principal dessa doença. Este artigo tem como objetivo estudar a maneira que este desequilíbrio da microbiota intestinal possa influenciar no desenvolvimento ou na exacerbação da síndrome do intestino irritável. A disbiose, que é a interrupção da relação simbiótica no intestino, gera a facilitação para a invasão e crescimento de espécies patogênicas no trato gastrointestinal levando ao desequilíbrio da homeostase do sistema imunológico e da barreira mucosa. Dessa forma, essa alteração das bactérias colonizadoras do intestino podem levar a uma alteração na barreira intestinal e na expressão de proteínas antimicrobianas levando a síndrome do intestino irritável. Diante disso, alguns probióticos vem sendo testados para o tratamento de síndrome do intestino irritável, visto que eles têm como objetivo a recomposição da microbiota e o reestabelecimento do equilíbrio intestinal.

Palavras-chave: Probióticos, Síndrome do intestino irritável, Microbiota.

\footnotetext{
Abstract: This review article sought to discuss the relationship of the intestinal microbiota in irritable bowel syndrome, which is one of the most common diseases of the gastrointestinal tract in the world and leads to changes in intestinal habits causing abdominal pain and discomfort. Its pathophysiology is still not well established, however, recent studies show that alteration of the intestinal microbiota may be the main trigger of this disease. This article aims to study how this imbalance in the intestinal microbiota can influence the

${ }^{1}$ Centro Universitário de Caratinga (UNEC), Caratinga - MG. *E-mail: mayralouresol@gmail.com

2 Faculdade de Medicina de Campos (FMC), Campos de Goytacazes - RJ.

${ }^{3}$ Faculdade Evangélica Mackenzie Paraná (FEMPAR), Curitiba - PR.

${ }^{4}$ Instituto Metropolitano de Ensino Superior (IMES), Ipatinga - MG.

${ }^{5}$ Faculdade Dinâmica do Vale do Piranga (FADIP), Ponte Nova - MG.

${ }^{6}$ Centro Universitário de Belo Horizonte (UniBH), Belo Horizonte - MG.

7 Universidade Católica de Pelotas (UCOel), Pelotas - RS.

8 Universidade Federal do Espírito Santo (UFES), Vitória - ES.

9 Universidade de Ribeirão Preto (Unaerp), Ribeirão Preto - SP.
} 
development or exacerbation of irritable bowel syndrome. Dysbiosis, which is the interruption of the symbiotic relationship in the intestine, facilitates the invasion and growth of pathogenic species in the gastrointestinal tract, leading to an imbalance in the homeostasis of the immune system and the mucous barrier. Thus, this change in colonizing bacteria in the intestine can lead to a change in the intestinal barrier and in the expression of antimicrobial proteins leading to irritable bowel syndrome. Therefore, some probiotics have been tested for the treatment of irritable bowel syndrome, since they aim to restore the microbiota and reestablish intestinal balance.

Keywords: Probiotics, Irritable bowel syndrome, Microbiota.

Resumen: Este artículo de revisión buscó discutir la relación de la microbiota intestinal en el síndrome del intestino irritable, que es una de las enfermedades del tracto gastrointestinal más comunes en el mundo y conduce a cambios en los hábitos intestinales que provocan dolor y malestar abdominal. Su fisiopatología aún no está bien establecida, sin embargo, estudios recientes han demostrado que los cambios en la microbiota intestinal pueden ser el principal desencadenante de esta enfermedad. Este artículo tiene como objetivo estudiar cómo este desequilibrio en la microbiota intestinal puede influir en el desarrollo o exacerbación del síndrome del intestino irritable. La disbiosis, que es la interrupción de la relación simbiótica en el intestino, facilita la invasión y el crecimiento de especies patógenas en el tracto gastrointestinal, provocando un desequilibrio en la homeostasis del sistema inmunológico y la barrera mucosa. Por tanto, este cambio en las bacterias colonizadoras del intestino puede provocar un cambio en la barrera intestinal y en la expresión de proteínas antimicrobianas que conducen al síndrome del intestino irritable. Por ello, se han probado algunos probióticos para el tratamiento del síndrome del intestino irritable, ya que tienen como objetivo restaurar la microbiota y restablecer el equilibrio intestinal.

Palabras clave: Probióticos, Síndrome del intestino irritable, Microbiota.

\section{INTRODUÇÃO}

A síndrome do intestino irritável (SII) é um distúrbio do trato gastrointestinal (GI), caracterizada por dor ou desconforto abdominal, alterações dos hábitos intestinais e características das fezes (LIU H, et al., 2017). O trato GI humano é habitado por um conjunto de micro-organismos conhecidos como microbiota intestinal (MI). Esta está amplamente envolvida em processos vitais, como desenvolvimento do sistema imunológico, manutenção da fisiologia do trato digestivo e digestão de carboidratos não digeridos. Dessa forma, alterações na sua estrutura e composição estão envolvidas no desenvolvimento de doenças sistêmicas crônicas e gastrointestinais, como a SII (BHATTARAI Y, et al., 2017).

Com prevalência de $11,2 \%$ em todo o mundo, a SII está associada a diminuição da produtividade no trabalho e redução da qualidade de vida dos pacientes. Sua causa ainda não está bem esclarecida, mas acredita-se estar relacionada a múltiplos mecanismos fisiopatológicos, incluindo aumento da permeabilidade visceral, resposta imune anormal e desregulação do eixo cérebro-intestino. Além disso, aproximadamente $84 \%$ dos pacientes com a SII relataram eventos de estresse e alimentos como principais gatilhos (SU H, et al., 2019).

Sabe-se também que a Ml é uma fonte de milhares de pequenas moléculas que podem desencadear as vias metabólicas e imunológicas do hospedeiro. Ela é dominada por 4 filos bacterianos, mas também inclui grande número de vírus, protozoários, fungos e archaea. Desse modo, o estudo do desenvolvimento e função da $\mathrm{Ml}$ é fundamental para a compreensão dos distúrbios gastrointestinais ao longo da vida (RASKOV H, et al., 2016; MARTINEZ K, et al., 2017).

Pesquisas no campo da Ml aumentaram, intensificando o conhecimento acerca de sua função no desenvolvimento de patologias e sua manutenção da saúde no organismo humano (PASSOS MCF e MORAES-FILHO JP, 2017). Tendo em vista essas questões, esse estudo objetiva-se de reunir nas 
bibliografias coletadas informações que relacionem o papel da MI na SII, a fim de estabelecer de que maneira o desequilíbrio da MI interfere na SII, tanto no seu desenvolvimento, quanto em sua exacerbação.

\section{REVISÃO BIBLIOGRÁFICA}

O organismo humano é hospedado por trilhões de micro-organismos que residem no trato gastrointestinal (TGI), cobrindo mais de $200 \mathrm{~m}^{2}$ de mucosa. O número deles é 10 vezes maior que o número de células do nosso corpo, além de terem 150 vezes mais genes que o genoma humano. A relação ecológica que os microorganismos mantêm com o organismo humano é chamada simbiose, esta, exerce influência na interação entre bactérias benéficas e células imunológicas do hospedeiro, levando à regulação dos genes humanos pró e anti-inflamatórios, estes sendo regulados positivamente e, aqueles, negativamente. Evidências crescentes têm mostrado que uma alteração permanente na composição ou função da microbiota pode alterar as respostas imunológicas, metabolismo, permeabilidade intestinal e motilidade digestiva, promovendo, então, um estado pró-inflamatório no TGI (RASKOV H, et al., 2016; PASSOS MCF e MORAES-FILHO JP, 2017).

Mantendo uma relação simbiótica, de vantagens mútuas com o organismo de um indivíduo, a MI auxilia na preservação de funções imunológicas, na absorção e digestão adequada de nutrientes, e nas funções metabólicas habituais. Do mesmo modo, ao realizar um efeito relevante na resposta imunitária humana, a MI também desempenha papel importante na preservação da imunidade intestinal e no amadurecimento dos tecidos linfoides do TGI (PASSOS MCF e MORAES-FILHO JP, 2017).

Dessa forma, a preservação da eubiose (relação simbiótica fisiológica) é essencial para manter a integridade do epitélio intestinal, criando uma barreira ideal para proteção contra patógenos, bem como para a captação de nutrientes. Já a disbiose (interrupção da relação simbiótica), refere-se às doenças microbianas que geram desequilíbrio no corpo ou dentro dele, e é mais comumente relatado em termos de alterações no TGI. Muitos fatores podem influenciar negativamente e gerar disbiose: uso de antibióticos, fatores psicológicos, estresse, radiação, peristaltismo alterado e mudanças na dieta. A disbiose torna favorável a invasão e crescimento de espécies patogênicas, interrompendo a homeostase do sistema imunológico e da barreira mucosa (RASKOV H, et al., 2016; MORAES-FILHO JP e QUIGLEY EMM, 2015; PASSOS MCF e MORAES-FILHO JP, 2017).

Pacientes portadores de SII possuem número maior de fatores de virulência presentes em sua MI, esses fatores participam na expressão de um maior potencial de patogenia das bactérias, através de diferentes modos de agir, como inibição da resposta imunológica ou aumento da adesão de bactérias à mucosa intestinal do hospedeiro (VILA AV, et al., 2018).

A disfunção da barreira intestinal pode estar relacionada ao distúrbio da $\mathrm{Ml}$, a depender das características dessa microbiota. Este é possivelmente um fator que contribui para a inflamação de baixo grau e uma maior chance de infecção no TGI, uma vez que a MI auxilia na expressão de mucinas; na redistribuição das zonas de oclusão interferindo, diretamente na barreira epitelial e também na expressão de proteínas antimicrobianas, como $\alpha$-defensinas e matripsina, que desempenham um papel importante na prevenção de infecções bacterianas patogênicas (FAN WT, et al., 2017).

A análise do gene 16s rRNA é o método mais comum para o estudo de microbioma, este, considerado o perfil genômico da microbiota (PITTAYANON R, et al., 2019; RASKOV H, et al., 2016). De acordo com o estudo de Duan R, et al. (2019), foi demonstrado que, com base em sequenciamento do 16S rRNA, existem mudanças relativamente consistentes na microbiota fecal para pacientes com SIl quando comparados com os grupos controles. No tocante aos dois maiores filos bacterianos que colonizam o intestino humano: Firmicutes e Bacteroidetes, os pacientes com SII mostraram uma maior abundância dos primeiros e diminuição dos últimos em comparação com os controles e, portanto, um aumento na razão de Firmicutes/Bacteroidetes (de aproximadamente 1,2-3,5 vezes). Além desses, os com SII mostraram aumento de Clostridia e Clostridiales e diminuição de Bacteroidia e Bacteroidales em níveis taxonômicos mais baixos. Além disso, Kerckhoffs APM, et al. (2009) fizeram o relato da utilização da técnica de PCR em tempo real para analisar a composição de Bifidobactérias em amostras de escova da mucosa duodenal de pacientes com SII, mostrando que a quantidade de $B$. catenulatum foi reduzida em comparação com controles. 
O estudo Giamarellos-Bourboulis E, et al. (2015) relatou alterações significativas de certos gêneros em análise de aspirados duodenais, englobando a super-representação de Escherichia/Shigella e Aeromonas e a sub-representação de Acinetobacter, Citrobacter, Microvirgula, Flavobacterium, Enhydrobacter, Weissella, Leuconostoc, Chryseobacterium e Lactococcus. No que diz respeito à microbiota da mucosa do jejuno proximal, foi relatado uma proporção maior de Firmicutes/Actinobacteria em pacientes com SIl em comparação com os controles saudáveis, além de maiores proporções de Streptococcus, Prevotella, Helicobacter, Brevibacterium e uma menor proporção de Neisseria em nível de gênero em pacientes com SII (CHUNG CS, et al., 2016). Em relação às alterações na microbiota da mucosa do cólon, pacientes com SII apresentaram contagens mais altas de Bacteroidaceae, já quanto à microbiota da mucosa do reto, pacientes com SII mostraram frequências aumentadas de Ochrobactrum e diminuídas de Faecalibacterium (DURBÁN A, et al., 2012; LIANG D, et al., 2018).

Em suma, determinadas condições de virulência são essenciais na explicação do benefício seletivo de bactérias que são muito patogênicas em indivíduos cuja Ml é saudável. Assim, alterações na constituição da MI podem levar ao desenvolvimento de SII, como o aumento da adesão de bactérias à mucosa, a maior secreção de toxinas, a mudança de composição da mucosa ou a própria disputa por recursos do hospedeiro com a microbiota. Então, os estudos que vêm sendo realizados até o momento se direcionam aos meios de patogenicidade das doenças TGI por meio de grupos específicos de bactérias (VILA AV, et al., 2018).

Visto que o desequilíbrio e a alteração da composição da MI contribuem para a patogênese da SII, recentemente tem sido instituída como terapêutica complementar a utilização de probióticos. Estes são definidos como micro-organismos vivos que auxiliam de forma positiva para com a saúde. Além disso, possuem papel fundamental no ambiente intestinal uma vez que ajustam o peristaltismo, estimulam a produção de muco pelas células caliciformes, auxiliam na manutenção da barreira intestinal e evitam a proliferação descontrolada de bactérias patogênicas (COSTA SAL, et al., 2020).

Vários estudos demonstraram que há melhoria dos sintomas relacionados à SII com a utilização de probióticos que possuem variedade de espécies bacterianas. Assim, os probióticos multiespécies demonstraram resultado superior quando comparados ao uso de placebo ou mesmo ao uso de probióticos com apenas uma espécie. Foi observada notável melhora da qualidade de vida dos portadores de SII bem como regularização da consistência das fezes e diminuição da presença de flatulência. Ressalta-se também que é importante direcionar o tipo de probiótico segundo os sintomas referidos pelos pacientes. Assim, notouse que para redução de dor e flatulência E. coli Nissle, Lactobacillus acidophilus ou Lactobacillus GG apresentam bom desempenho, já para casos de constipação utilizou-se E. coli Nissle, Bifidobacterium animalis ou L-casei Shirota enquanto que em quadros diarreicos os probióticos escolhidos possuíam Lactobacillus, mistura de bactérias do ácido láctico ou Bifidobacterium (COSTA SAL, et al., 2020).

Apesar dos potenciais benefícios terapêuticos do uso de probióticos na SII, ainda se encontram divergências relativas a tal prática no meio científico. Em vista disso, uma revisão sistemática com metanálise publicada em 2018, analisou estudos com resultados destoantes. Dentre esses estudos, observou-se que em 21 estudos randomizados controlados foram utilizados probióticos combinados e como consequência houve relevante ação sobre a persistência dos sintomas da SII, porém tais estudos possuíam muitos fatores dissemelhantes entre si. Além disso, observou-se que em 3 estudos houve a utilização probiótica combinada de Lactobacillus paracasei ssp paracasei F19, Lactobacillus acidophilus La5 e Bifidobacterium lactis Bb12 comparando-os com placebo e concluiu-se que não houve diferença na constância da sintomatologia da doença entre os grupos que utilizaram placebo com os grupos que fizeram uso de probiótico. Já em outros oitos estudos, totalizando quase 900 pacientes, em que se analisou a aplicabilidade do Lactobacillus verificouse que não houve superioridade em relação ao uso de placebo e que novamente havia heterogeneidade entre os estudos (FORD AC, et al., 2018).

Apesar disso, ainda houve outros estudos que se demonstraram positivos, dessa forma quando ocorreu a utilização de Bifidobacterium, Escherichia e Streptococcus faecium houve certa superioridade em relação ao placebo. Outra revisão sistemática com metanálise divulgada em 2019, corroborou com alguns achados de estudos anteriores ao demonstrar que em 17 estudos randomizados controlados o uso de probióticos 
proporcionou significativa melhora dos sintomas globais da SII, porém esses estudos também se apresentavam inomogêneos. Nos probióticos analisados nessa metanálise, observou-se que em vários estudos controlados randomizados os produtos que possuíam espécies de Lactobacillus aliviaram flatulência e dor abdominal, os que possuíam Saccharomyces diminuíram a sensação de distensão abdominal, enquanto que a utilização de Bifidobacterium melhorou a questão de urgência fecal (FORD AC, et al., 2018; ASHA MZ e KHALIL SFH, 2019).

Em um estudo de metanálise em rede de 2019, foi avaliada a eficácia de protocolos probióticos contendo a combinação de Lactobacillus e Bifidobacterium que são os principais encontrados em produtos probióticos, pois em alguns estudos verificou-se decréscimo na quantidade desses gêneros bacterianos em pacientes portadores de SII. Assim pela utilização dessa mistura probiótica notou-se que ocorreram significativos resultados na melhora dos sintomas gerais da SII quando se comparou com a utilização de probióticos de apenas uma espécie. Os efeitos benéficos do uso de Lactobacillus e Bifidobacterium são devido ao fato de essas bactérias secretarem bactericinas, auxiliarem na modulação do sistema imune, entre outros efeitos. Além disso, observou-se que uma menor dosagem de probióticos apresentou maior eficácia na redução de sintomas do que a utilização de uma superdosagem, uma vez que isso pode ocasionar maior competição entre as cepas bacterianas atrapalhando a sobrevivência de cada uma, pode também agravar a disbiose dos pacientes com SII e até causar desconforto intestinal pelo excesso de fermentação, especialmente se houver Lactobacillus e Bifidobacterium, os quais realizam digestão de carboidratos (RODIÑO-JANEIRO BK, et al., 2018; LIANG D, et al., 2019).

Também nessa metanálise, foi preconizada como dose probiótica eficaz para a SII a dosagem de $10^{9} \sim 10^{10}$ UFC (unidades formadoras de colônia) / dia. Foi abordada a utilização do protocolo DUO, o qual é composto por 7 tipos de bactérias, a saber: Lactobacillus acidophilus, Lactobacillus plantarum, Lactobacillus rhamnosus, Bifidobacterium breve, Bifidobacterium lactis, Bifidobacterium longum e Streptococcus thermophilus, e observou-se que a terapêutica com esse protocolo apresentou a maior eficácia entre os protocolos analisados para o subtipo diarreico da SII. Já o protocolo Pro (Bifidobacterium lactis Bb12, Lactobacillus acidophilus La5, Lactobacillus delbruecki subsp bulgaricus LBY-27, and Streptococcus thermophilus STY-31) foi mais efetivo para o subtipo indiferenciado da SII (LIANG D, et al., 2019).

Analisando-se os resultados de diversos estudos realizados pode-se observar que o uso de probióticos apresenta eficácia e segurança e que a utilização de multiespécies foi superior ao uso de placebo e de espécies únicas (MORAES-FILHO JP e QUIGLEY EMM, 2015; OOI SL, et al., 2019; DALE HF, et al., 2019).

Os probióticos através de diversos mecanismos como, por exemplo, contribuir para a integridade da barreira intestinal e competir com bactérias patogênicas, auxiliam na modulação da MI dos portadores de SII propiciando alívio dos sintomas (ASHA MZ e KHALIL SFH, 2019; COSTA SAL, et al., 2020)

Vários estudos relataram que a terapêutica probiótica auxiliou nos sintomas gerais, outros relataram alívio de flatulência, melhoria na consistência fecal, já em relação à diminuição da distensão e dor abdominal houve divergência entre os estudos. Portanto, nota-se que apesar de os probióticos demonstrarem-se benéficos na SII são necessários mais estudos futuros para que haja a padronização do tempo de tratamento e da dosagem. Também é necessário que haja consenso entre quais as espécies bacterianas são mais eficazes para cada sintoma da SII, visto que há grande variedade tanto na combinação probiótica quanto nos subtipos de apresentação clínica da SII (FORD AC, et al., 2018; DALE HF, et al., 2019; COSTA SAL, et al., 2020).

Uma das possibilidades terapêuticas para SII que também vem sendo estudada nos últimos anos é o Transplante Fecal de Microbiota (TFM), desde que o uso de TFM foi considerado eficaz para tratar infecções por Clostridium difficile (BENNO P, et al., 2019).

Dessa forma, através de estudos comparativos foi evidenciada uma diferença significativa entre a microbiota fecal de portadores da SII e a microbiota de pessoas que possuíam um intestino saudável, diferindo de forma significativa principalmente em relação a composição da microbiota, de forma que o filo Actinobacteria foi encontrada em menor quantidade nos pacientes com SIl que no grupo de indivíduos saudáveis (MAZZAWI T, et al., 2019). 
O TFM consiste em uma coleta de fezes de indivíduos saudáveis que após análises são transplantadas para portadores da SII através de uma gastroscopia duodenal. Cerca de 3 semanas após o procedimento de TFM a maioria dos portadores da SII costumam apresentar uma microbiota normalizada, ou seja, com melhora dos sintomas e consequente melhora na qualidade de vida dos pacientes. Nota-se então, que o TFM altera o intestino disbiótico de pacientes portadores de SII, sendo que essa diminuição dos sintomas costuma persistir por cerca de 28 semanas (MAZZAWI T, et al., 2019).

Dentre os estudos que questionaram indiretamente a eficácia do TFM , tem-se um estudo comparativo entre 23 portadores da SII e 24 pessoas saudáveis, que analisou indivíduos com gênero e idades semelhantes, encontrando Firmicutes, Bacteroidetes e Actinobacteria como filos predominantes em ambos os grupos (saudáveis e com SII) sendo que a diferença entre a microbiota dos grupos foi considerada não significativa, o que por sua vez não justificaria a realização do TFM, entretanto o próprio estudo traz como limitações o número de amostras e a seleção realizada (MAHARSHAK N, et al., 2018).

Um dos estudos que considerou eficaz o TFM para pacientes com SII, observou melhora nos sintomas de portadores de SII que apresentavam predominantemente: diarreia, constipação ou sintomas mistos, relatando também alguns dos efeitos adversos do TFM, mas que foram restritos aos primeiros dois dias após o procedimento, sendo que os efeitos adversos consistiram em aumentar de forma significativa: constipação, diarreia, naúseas e dor abdominal. Outro efeito adverso foi que 2 dos 165 pacientes avaliados desenvolveram diveticulose em cerca de 2 a 3 meses após o TFM, sofrendo com diverticulites recorrentes nos meses seguintes (EL-SALHY M, et al., 2019).

Outro estudo que sugere a eficácia do TFM realizou-o a partir de um único doador que não possuía histórico de moléstias infecciosas gastrointestinais, geniturinárias ou pulmonares e havia sido pouco exposto a antibióticos previamente, dessa forma os 50 portadores da SIl selecionados eram 25 homens e 25 mulheres. Após o TFM 32 tiveram reduções significativas dos sintomas, no entanto 20 dos portadores de SII tiveram apenas uma passageira redução de sintomas após o TFM (BENNO P, et al., 2019).

Algumas razões que justificam resultados ineficazes do TFM seriam basicamente devido: à incompatibilidade da flora do doador e receptor, quantidade insuficiente de bactérias instiladas no momento da gastroscopia, etiologia da SII de determinado paciente não ter relação com a disbiose intestinal e o modo de administração não ser considerado ideal (BENNO P, et al., 2019).

No entanto, foi demonstrado que o TFM tem se tornado uma opção terapêutica de baixo custo e muito eficaz para a SII por ser capaz de alterar a disbiose intestinal dos portadores da SII (WEN W, et al., 2018).

\section{CONSIDERAÇÕES FINAIS}

Através da análise dos dados supracitados, conclui-se que alterações nas funções da microbiota intestinal gera efeito na saúde e um impacto na patogenicidade de doenças gastrointestinais. Desse modo, os avanços em pesquisas da SII resultaram em análises do uso de probióticos multiespécies demonstrando-se benéficos. Essas descobertas têm apresentado melhora dos sintomas com a combinação de Lactobacillus $e$ Bifidobacterium, visto que comparados ao uso de placebo ou probióticos de apenas uma espécie possuem melhores resultados. No entanto, apesar desses avanços, ainda há muito a ser descoberto em relação a MI e suas implicações na saúde, uma vez que se encontra envolvida em vias metabólicas e imunológicas do hospedeiro.

\section{REFERÊNCIAS}

1. ASHA MZ, KHALIL SFH. Efficacy and Safety of Probiotics, Prebiotics and Synbiotics in the Treatment of Irritable Bowel Syndrome A systematic review and meta-analysis. Sultan Qaboos University Medical Journal, 2019; 20(1):1324.

2. BENNO P, et al. Therapeutic potential of an anaerobic cultured human intestinal microbiota, ACHIM, for treatment of IBS. Best Practice \& Research: Clinical Gastroenterology, 2019; 40-41:101607. 
3. BHATTARAI Y, et al. Irritable bowel syndrome: a gut microbiota-relateddisorder?. Am J Physiol Gastrointest Liver Physiol, 2017; 312(1): 52-62.

4. COSTA SAL, et al. Uso de probióticos no tratamento de pacientes com síndrome do intestino irritável. Brazilian Journal of Health Review, 2020;3(4):11047-11060.

5. CHUNG CS, et al. Differences of microbiota in small bowel and faeces between irritable bowel syndrome patients and healthy subjects. Scandinavian Journal of Gastroenterology, 2016; 51(4):410-419.

6. DALE HF, et al. Probiotics in Irritable Bowel Syndrome: An Up-to-Date Systematic Review. Nutrients, 2019; 11(9):2048.

7. DUAN R, et al. Alterations of Gut Microbiota in Patients With Irritable Bowel Syndrome Based on 16S rRNA-Targeted Sequencing: A Systematic Review. Clinical and Translational Gastroenterology, 2019; 10(2):1-12.

8. DURBÁN A, et al. Structural alterations of faecal and mucosa-associated bacterial communities in irritable bowel syndrome: Microbial diversity in irritable bowel syndrome. Environmental Microbiology Reports,2012; 4(2):242-247.

9. EL-SALHY M, et al. Efficacy of faecal microbiota transplantation for patients with irritable bowel syndrome in a randomised, double-blind, placebo-controlled study. Gut, 2019;69:859-867.

10. FAN WT, et al. Close association between intestinal microbiota and irritable bowel syndrome. European Journal of Clinical Microbiology \& Infectious Diseases, 2017; 36(12):2303-2317.

11. FORD AC, et al. Systematic review with meta-analysis: the efficacy of prebiotics, probiotics, synbiotics and antibiotics in irritable bowel syndrome. Alimentary Pharmacology \& Therapeutics, 2018; 48(10):1044-1060.

12. GIAMARELLOS-BOURBOULIS E, et al.Molecular assessment of differences in the duodenal microbiome in subjects with irritable bowel syndrome. Scandinavian Journal of Gastroenterology, 2015; 50(9):1076-1087.

13. KERCKHOFFS APM, et al. Lower Bifido bacteria counts in both duodenal mucosa-associated and fecal microbiota in irritable bowel syndrome patients. World Journal of Gastroenterology, 2009; 15(23):2887-2892.

14. LIANG D, et al. Efficacy of different probiotic protocols in irritable bowel syndrome A network meta-analysis. Medicine (Baltimore), 2019; 98 (27):16068.

15. LI G, etal.Involvement of shared mucosal-associated microbiota in the duodenum and rectum in diarrhea-predominant irritable bowel syndrome: Shared microbiota in IBS with diarrhea. Journal of Gastroenterology and Hepatology,2018; 33(6):1220-1226.

16. LIU H, et al. Altered molecular signatureof intestinal microbiota inirritable bowel syndrome patients compared with healthy controls: a systematic review and meta-analysis. DigestiveandLiverDisease,2017; 49(4): 331-337.

17. MAHARSHAK N, et al. Fecal and mucosa-associated intestinal microbiota in patients with diarrhea-predominant irritable bowel syndrome. Digestive Diseases and Sciences, 2018;63(7): 1890-1899.

18. MARTINEZ K, et al. Microbial metabolites in healthanddisease: Navigatingtheunknown in searchoffunction. J. Biol. Chem, 2017; 292(21): 8553- 8559.

19. MAZZAWI T, et al. Clinical response to fecal microbiota transplantation in patients with diarrhea-predominant irritable bowel syndrome is associated with normalization of fecal microbiota composition and short-chain fatty acid levels. Scandinavian Journal of Gastroenterology, 2019; 54(6):690-699.

20. MORAES-FILHO JP, QUIGLEY EMM. The intestinal microbiota and the role of probiotics in irritable bowel syndrome: a review. Arquivos de Gastroenterologia, 2015; 52(4):331-338.

21. OOI SL, et al. Probiotics, prebiotics, and low FODMAP diet for irritable bowel syndrome - What is the current evidence?.ComplementaryTherapies In Medicine, 2019; 43: 73-80.

22. PASSOS MCF, MORAES-FILHO JP. Intestinal microbiota in digestivediseases. Arq. Gastroenterol. 2017; 54(3):255262.

23. PITTAYANON R. et. al. Gut Microbiota in Patients With Irritable Bowel Syndrome-A Systematic Review. Gastroenterology, 2019; 157(1): 97-108.

24. RASKOV H, et al. Irritable bowel syndrome, the microbiota and thegut-brainaxis. Taylor e Francis, 2016; 7(5): 365368.

25. RODIÑO-JANEIRO BK, et al. A Review of Microbiota and Irritable Bowel Syndrome. Future in Therapies. Advances in Therapy, 2018; 35 (3):289-310.

26. SU H, et al. EffectsofLow-FODMAPS Diet on Irritable Bowel Syndrome Symptoms and Gut Microbiome. Gastroenterology Nursing, 2019; 42(2): 150-158.

27. VILA AV, et al. Gut microbiota composition and functional changes in inflammatory bowel disease and irritable bowel syndrome. Science Translational Medicine, 2018; 10(472):1-11. 